\title{
Importância dos Parâmetros Seminais nos Resultados de Inseminação Intra-uterina
}

\author{
Importance of Seminal Parameters for Intrauterine Insemination Outcomes
}

Edson Borges Junior, Lia Mara Rossi, Cláudia Chagas Rocha, Fernando Calabresi, Wagner Camargo Bussato, Assumpto Iaconelli Jr.

\begin{abstract}
RESUMO
Objetivos: analisar a relevância dos parâmetros seminais nos resultados dos ciclos de inseminação intra-uterina (IIU) em pacientes com causa masculina de infertilidade e salientar o caráter prognóstico de cada parâmetro para o sucesso da técnica.

Métodos: duzentos e trinta e nove ciclos de IIU (155 casais) foram analisados durante periodo de 15 meses. Todos as pacientes foram submetidas à indução da ovulação de acordo com protocolos do I Consenso Brasileiro de Indução de Ovulação. A análise seminal foi realizada de acordo com os critérios da Organização Mundial da Saúde (OMS) para parâmetros como concentração e motilidade e critério estrito de Kruger para avaliação da morfologia. O preparo da amostra para IIU foi realizado por meio de gradientes descontinuos de densidade (ISolate ${ }^{\circledR}$ ). Foram formados dois grupos de pacientes de acordo com o resultado de gestação: grupo G gestação positiva e grupo NG - gestação negativa.

Resultados: não houve diferença significativa nos valores obtidos relacionados à concentração total de espermatozóides $/ \mathrm{mL}$ de sêmen, motilidade total, motilidade de formas progressivas antes e depois do processamento seminal. Quando a morfologia estrita de Kruger foi comparada entre os dois grupos, diferenças significativas foram encontradas (grupo $G=10,6 \%$ de formas normais; grupo $N G=6,4 \%$ de formas normais; $p<0.05$ ) quando o número de espermatozóides/ $\mathrm{mL}$ inseminados foi superior a 15 milhões/ $\mathrm{mL}$, a taxa de gravidez foi significantemente maior. Conclusões: tanto a morfologia estrita de Kruger como o número de espermatozóides inseminados parecem ser fatores prognósticos positivos para o estabelecimento da gestação, devendo ser salientados na investigação do homem infértil.
\end{abstract}

PALAVRAS-CHAVE: Inseminação artificial. Infertilidade. Sêmen: análise.

Introdução

O conhecimento do transporte de espermatozóides através do trato genital feminino na espécie humana ainda permanece limitado. No entanto, sabe-se que, dos milhões de espermatozóides ejaculados e depositados na vagina, somente alguns serão capazes de alcançar o canal cervical, penetrar no muco cervical, migrar através da luz do útero e avançar em direção à tuba uterina. Somente cerca de 100 espermatozóides móveis conseguirão, de fato, entrar em contato com o oócito ${ }^{1}$.

Fertility - Centro de Fertilização Assistida - São Paulo Correspondência:

Lia Mara Rossi

Avenida Brigadeiro Luís Antonio, 4251

01402-001 - São Paulo - SP

Fone/fax: + 55 (11) 3885-9858 / 3887-2628

e-mail: lia@fertility.com.br
Existem certas causas de infertilidade, nas quais o processo de aproximação dos gametas está alterado em algumas de suas etapas. Para estes casos, técnicas de baixa complexidade em reprodução assistida - como a inseminação intrauterina (IIU) - são recomendadas ${ }^{2}$.

Entre as indicações clássicas para IIU podemos salientar: anomalias congênitas do trato genital, disfunções eréteis (psicológica ou orgânica), ejaculação retrógrada e/ou disfunções sexuais femininas (vaginismo), ausência de muco ou presença de muco hostil (fator cervical). Quanto às causas seminais podemos citar: oligozoospermia e/ ou astenozoospermia (fator masculino com causa seminal leve ou moderada), uso de sêmen de doador em procedimentos com mulheres solteiras ${ }^{3}$ (Conselho Federal de Medicina, Resolução 1.358/ 92) ou uso de sêmen criopreservado previamente à vasectomia e tratamentos de radioterapia ou quimioterapia em pacientes portadores de neoplasias ${ }^{4}$. 
Apesar de ser método efetivo e consagrado no tratamento da infertilidade, sua eficácia apresenta significativa redução após várias tentativas sem sucesso, restringindo-se a 3 ou 4 ciclos $^{5}$.

Independentemente da indicação para IIU, o sêmen deverá ser previamente analisado com o intuito de selecionar amostra com concentração adequada de espermatozóides exibindo motilidade linear progressiva. Além destas condições, a morfologia dos espermatozóides da amostra é também verificada e contribui para a indicação desta técnica.

Principalmente nos casos em que a IIU é indicada em virtude da presença de alterações seminais leves ou moderadas, ainda existe dúvida na literatura relacionada à importância dos parâmetros seminais sobre as taxas de sucesso desta técnica e aos valores superiores ou limitrofes que fazem parte do critério de inclusão para a indicação desta técnica. Uma meta-análise revela que casais com infertilidade de causa masculina exibem metade da taxa de gestação conseguida em outros casais com outras causas de infertilidade ${ }^{6}$. Em contraste, outro estudo retrospectivo randomizado concluiu que a IIU é igualmente eficiente em casais com causas masculinas ou idiopáticas de infertilidade ${ }^{7}$. Conclusões opostas, no entanto, podem ser resultado das diferentes definições de "fator masculino" para causas de infertilidade e a gravidade da causa masculina pode ser fator que interfere nos resultados.

Procuramos neste estudo, a partir de análise retrospectiva dos ciclos de IIU realizados em nosso serviço por periodo de 15 meses, avaliar a influência dos parâmetros seminais (concentração total de espermatozóides $/ \mathrm{mL}$ de sêmen, motilidade total, motilidade de formas progressivas, morfologia e número de espermatozóides/mL inseminados) nos resultados deste procedimento realizado em casais com causa masculina de infertilidade.

\section{Pacientes e Métodos}

Foram estudados retrospectivamente 438 casais atendidos no período de 20 de outubro de 2001 a 20 de janeiro de 2003. Procedeu-se à anamnese dos casais visando valorizar antecedentes que pudessem interferir na fertilidade, tais como doença pélvica inflamatória, cirurgias ginecológicas, pélvicas ou do trato genital masculino, uso de drogas, doenças sexualmente transmissíveis e doenças genéticas familiares, entre outras. Após exame físico geral do casal, aplicou-se a propedêutica básica específica em reprodução humana assistida: análise seminal completa para a avaliação do status funcional dos testículos; histerossalpingografia e/ou videolaparoscopia para avaliação da cavidade uterina, das tubas uterinas e região pélvica; teste pós-coito - SimsHuhner - para avaliação do fator cervical e, finalmente, a avaliação do funcionamento dos ovários pela ultra-sonografia seriada (para análise de desenvolvimento folicular) e dosagens de FSH e LH no terceiro dia do ciclo menstrual.

A partir dos resultados destes exames, foram selecionados 155 casais que atenderam aos critérios de inclusão, a saber: concordância em participar do estudo, ausência do fator feminino como causa de infertilidade (cavidade uterina normal; ovário funcionante, ou seja, aquele capaz de produzir folículos e oócitos (ciclos ovulatórios); pelo menos tuba pérvia e supostamente saudável para migração de espermatozóides e embrião) e concentração mínima de espermatozóides móveis progressivos pós-processamento de 5 milhões $/ \mathrm{mL}$.

A concordância em participar do estudo foi obtida por meio de consentimento informado datado e assinado pelos pacientes e aprovado pelo Conselho de Ética do Hospital e Maternidade Santa Joana, em São Paulo, tendo sido excluídos aqueles que não concordavam em participar.

A amostra de sêmen foi coletada por masturbação após 3-5 dias de abstinência sexual e foi submetida ao protocolo rotineiro para análise seminal, segundo normativa da Organização Mundial da Saúde ${ }^{8}$. Parâmetros macroscópicos como cor, volume, $\mathrm{pH}$, viscosidade e liquefação foram analisados, mas não comparados entre os grupos. Depois de liquefeita, a amostra foi mantida a $37^{\circ} \mathrm{C}$ por 30 minutos e, após este período, $10 \mu \mathrm{L}$ foram retirados e transferidos para câmara de contagem tipo Makler ou microcell para cálculo da concentração e motilidade espermática, a saber: grau A - motilidade rápida e progressiva; $\mathrm{B}$ - motilidade lenta e progressiva; $\mathrm{C}$ - motilidade não progressiva, e D - imóvel. Para análise morfológica foi utilizada a coloração verde-brilhante e/ou Papanicolaou modificado, sendo os espermatozóides classificados pelo critério descrito por Kruger et al. ${ }^{9}$ em 1986, em objetiva de imersão em microscópio de luz convencional. Foram considerados normais os espermatozóides com cabeça entre 5 e $6 \mathrm{~mm}$ de comprimento, 2,5 a 3,5 $\mathrm{mm}$ de diâmetro, 40 a 70\% de acrosoma, sem defeitos de cauda ou peça intermediária.

Para o preparo do sêmen foram utilizados neste estudo gradientes descontínuos de densidade (ISolate ${ }^{\circledR}$ ) com duas camadas: 0,5 mL com 95 e $47,5 \% \mathrm{v} / \mathrm{v}$ ISolate ${ }^{\circledR}$ ou 3,0 mL com 72 e $36 \% \mathrm{v} / \mathrm{v}$ ISolate $^{\circledR}$. Em tubo cônico foi depositado o gradiente inferior e por cima deste, o superior. Cuidadosamente sobre estes foi depositado 1,0 mL do sêmen, procedeu-se à centrifugação e o precipitado contendo os espermatozóides foi retirado. Após 
ressuspensão em meio de cultura, novas análises de concentração e motilidade foram realizadas. O material foi mantido em incubadora $\left(37^{\circ} \mathrm{C}\right)$ até o momento da IIU.

Os 155 casais perfizeram um total de 230 ciclos de IIU, nos quais procedeu-se à estimulação ovariana com a utilização de FSH recombinante iniciado no $3^{\circ}$ dia do ciclo menstrual, nas concentrações de 75 a $150 \mathrm{UI}^{2}$. Foi realizado o monitoramento por meio de ultra-sonografia transvaginal (USGTV), a partir do $6^{\circ}$ dia de uso da gonadotrofina, em dias alternados.

Para deflagrar a eclosão folicular, 10.000 UI de gonadotrofina coriônica humana (hCG) foram administradas via intramuscular, quando pelo menos um folículo maduro estava presente (diâmetro médio de $\geq 18 \mathrm{~mm}$ ou diâmetro máximo entre 18 e $20 \mathrm{~mm}$ pela USGTV). Além deste parâmetro, a observação do complexo cumulus corona intrafolicular, assim como o aspecto trilaminar do endométrio e o canal cervical aberto foram outros indicios de iminência de ovulação. A IIU foi realizada, em média, 36 a 40 horas após a administração do hCG. O procedimento de IIU foi sempre realizado por profissional habilitado e especializado nas técnicas de reprodução humana assistida.

Em todos os pacientes foi utilizado o cateter TOM-CAT (Sherwood Medical St. Louis, MO, USA), sem acompanhamento ultra-sonográfico.

$\mathrm{Na}$ manhã seguinte à IIU, era observado o desaparecimento ou a diminuição do folículo dominante, aumento da ecogenicidade intrafolicular e aparecimento de líquido livre na região retrouterina, sugerindo a ocorrência da ovulação. Nos casos de permanência dos folículos dominantes, a IIU era repetida cerca de 60 horas após a administração de hCG.

Os pacientes foram divididos em dois grupos, a saber: gestação clínica (saco gestacional e batimentos cardiacos fetais) comprovada (grupo G) e não ocorrência de gestação (grupo NG).

Para analisar estatisticamente os resultados obtidos no presente estudo, utilizaram-se percentagem, média, desvio padrão, o teste $t$ de Student e o teste exato de Fisher, com nivel de significância de $5 \%(\mathrm{p}<0,05)$.

\section{Resultados}

A média de ciclos por paciente, neste estudo, foi de 1,5 . Noventa e nove pacientes $(63,9 \%)$ submeteram-se ao procedimento apenas $1 \mathrm{vez} ; 39$ pacientes $(25,2 \%)$, duas vezes; 15 pacientes $(9,6 \%)$, três vezes e 2 pacientes (1,3\%), quatro vezes. Em 6,9\% dos pacientes $(n=11)$, houve a necessidade de re- petição do procedimento na manhã seguinte, em virtude da não ocorrência de ovulação. Entre as indicações para a IIU estiveram presentes neste grupo de estudo: fator masculino (38,6\%), infertilidade sem causa aparente $(32,9 \%)$, sêmen de doador $(18,6 \%)$, fator ovariano $(7,1 \%)$, fatores femininos e masculinos combinados $(1,4 \%)$ e ovários policísticos $(1,4 \%)$.

Do total de 155 pacientes, gestação clínica (grupo G) foi diagnosticada em 38 pacientes (39 ciclos), fornecendo uma taxa de gravidez por ciclo e por paciente de 16,9 e $24,5 \%$, respectivamente. Em 97,4\% dos casos (37 pacientes) a gestação ocorreu na primeira tentativa.

$\mathrm{O}$ número médio de folículos por paciente, detectados pela ultra-sonografia, foi de 1,9 $\pm 1,7$ vs 1,6 $\pm 2,0$ (grupos G e NG, respectivamente), sem diferença estatística ( $p=0.093)$.

Não houve diferença significativa entre a idade das mulheres nos grupos G e NG (34,2 $\pm 2,6$ us $34,1 \pm 2,8$, respectivamente, $p=0,081$ ) e dos homens $(42,1 \pm 3,2$ vs 43,4 $\pm 2,8$, grupos $\mathrm{G}$ e $\mathrm{NG}$, respectivamente, $\mathrm{p}=0,074)$.

Quando se analisou a concentração espermática do sêmen fresco dos 155 pacientes estudados, nenhuma diferença significativa foi encontrada entre os grupos G e NG. Também em relação à motilidade total $(\mathrm{A}+\mathrm{B}+\mathrm{C})$ e motilidade progressiva $(\mathrm{A}+\mathrm{B})$ não foram observadas diferenças significativas (Tabela 1).

Em relação à morfologia espermática, diferenças significativas foram observadas. A média de formas normais encontradas nas amostras do grupo $\mathrm{G}$ foi significativamente diferente da média de formas normais do grupo NG $(10,6$ vs $6,4 \%$, respectivamente, $\mathrm{p}=0,028$ ) (Tabela 1 ).

Tabela 1 - Análise comparativa entre alguns parâmetros seminais em amostras a fresco.

\begin{tabular}{|c|c|c|}
\hline & Grupo G & Grupo NG \\
\hline Ciclos/pacientes & $39 / 38$ & $200 / 117$ \\
\hline $\begin{array}{l}\text { Concentração final } \\
\text { de espermatozóides (x } 10 \text { 6/mL) }\end{array}$ & $68,8 \pm 29,2$ & $63,2 \pm 26,8$ \\
\hline $\begin{array}{l}\text { Percentagem de espermatozóides } \\
\text { móveis após o processamento }\end{array}$ & $55 \pm 12,2$ & $51 \pm 14,1$ \\
\hline $\begin{array}{l}\text { Porcentagem de espermatozóides } \\
\text { móveis progressivos }\end{array}$ & $37 \pm 8,8$ & $34 \pm 9,7$ \\
\hline $\begin{array}{l}\text { Morfologia estrita de } \\
\text { Kruger (\% formas normais) }\end{array}$ & $10,6 \pm 2,1^{*}$ & $6,4 \pm 2,2$ \\
\hline
\end{tabular}

$\mathrm{Na}$ análise do sêmen após o processamento, resultados muito semelhantes foram obtidos nos grupos G e NG, em relação a concentração de espermatozóides, motilidade total $(\mathrm{A}+\mathrm{B}+\mathrm{C}) \mathrm{e}$ motilidade progressiva $(A+B)$, sugerindo valores não significativamente diferentes (Tabela 2). 
Tabela 2 - Análise comparativa entre alguns parâmetros seminais em amostras pós-processamento

\begin{tabular}{lcc}
\hline & Grupo G & Grupo NG \\
\hline Ciclos/pacientes & $39 / 38$ & $200 / 117$ \\
$\begin{array}{l}\text { Concentração final de } \\
\text { espermatozóides (x 10 6/mL) }\end{array}$ & $78,2 \pm 31,8$ & $72,2 \pm 34,3$ \\
$\begin{array}{l}\text { Percentagem de espermatozóides } \\
\text { móveis após o processamento }\end{array}$ & $89,1 \pm 24,3$ & $88,2 \pm 21,2$ \\
$\begin{array}{l}\text { Porcentagem de espermatozóides } \\
\text { móveis progressivos }\end{array}$ & $77,0 \pm 12,0$ & $76,3 \pm 14,1$ \\
\hline
\end{tabular}

Dados apresentados como média + desvio padrão.

Em relação ao número de espermatozóides inseminados, foi possivel notar taxa de gestação significativamente maior quando o número de espermatozóides inseminados foi maior que $15 \times 10^{6} / \mathrm{mL}$ (Tabela 3 ).

Tabela 3 - Relação entre o número de espermatozóides inseminados e a ocorrência de gestação.

Número de espermatozóides inseminados Taxa de gestação

$-10 \times 10^{6}$

$3,2^{\mathrm{a}}$

$>10-15 \times 10^{6}$

$10,2^{b}$

$>15-20 \times 10^{6}$

$25,0^{c}$

$>20 \times 10^{6}$ $21,8^{c}$

a,b $p>0,05 ;{ }^{a, c} e^{b, c} p<0,05$ (teste $t$ de Student)

\section{Discussão}

Como parte integrante das técnicas de reprodução assistida, a inseminação intra-uterina propicia boa terapêutica que pode ser indicada para um grupo seleto de casais.

Neste trabalho procuramos analisar o impacto da qualidade da amostra seminal nos resultados de IIU, como o número de espermatozóides inseminados e alterações nos parâmetros seminais, nos casos sem causas femininas de infertilidade. No entanto, o sucesso em ciclos de IIU depende de vários outros fatores isolados e/ou combinados, entre eles: a idade materna, tempo e etiologia da infertilidade, protocolos de estimulação ovariana e número de tentativas de $\mathrm{IIU}^{5,10-13}$.

Obtivemos, neste estudo, taxa de gestação de 16,9 e $24,5 \%$ (respectivamente, por ciclo e por paciente) após estimulação ovariana controlada, o que parece exibir efeito positivo sobre as taxas de gestação ${ }^{14}$. A literatura mostra que as taxas médias de gestação por ciclo no ciclo natural ficam ao redor de $3,1 \%$, sendo $7,7 \%$ com o uso de citrato de clomifeno e $13,2 \%$ com as gonadotrofinas ${ }^{15}$. A partir deste estudo, temos empregado FSH recombinante a partir do $3^{\circ}$ dia do ciclo menstrual, nas dosagens de 75 a 150 UI, com monitoramento feito pela ultra-sonografia seriada a partir do $6^{\circ}$ dia, com emprego de $\mathrm{FSH}$ recombinante diariamente ajustando as dosagens de acordo com o ultra-som. Para deflaglar a eclosão folicular, utilizamos $10.000 \mathrm{UI}$ de hCG via intramuscular, quando pelo menos um folículo maduro estiver presente (diâmetro médio maior ou igual a $18 \mathrm{~mm}$ pelo ultra-som).

A escolha pelo FSH recombinante, e não pelo urinário, é justificada pela ausência de proteínas adversas, visto que é obtido por tecnologia altamente especializada de engenharia genética, pela ausência de hormônio luteinizante $(\mathrm{LH})$, devido ao alto teor de purificação, e por causa da fácil administração e absorção subcutânea ${ }^{16-18}$.

Um protocolo mais agressivo deve ser indicado nos casos de piores prognósticos, nos quais a idade materna, as alterações seminais e o número de tentativas com IIU sem gestação devem ser parâmetros considerados.

Em relação ao número de tentativas de IIU com indução de ovulação, a literatura sugere três ciclos de IIU como número ótimo para a obtenção de boas taxas de gestação, com posterior encaminhamento para fertilização in vitro ${ }^{5,18,19}$. Em nossos resultados, a média de ciclos por paciente foi de 1,5 e em apenas $6,9 \%$ dos casais houve a necessidade de repetir o ciclo no dia seguinte.

Observamos, neste estudo, que a maior parte dos parâmetros seminais comumente analisados para a indicação da técnica de IIU parecem não interferir nos resultados de gestação, desde que estejam dentro dos limites sugeridos pela OMS para a indicação da técnica.

Dickey et al. ${ }^{13}$, após estudo com 4.056 ciclos de IIU, concluíram que casais com parâmetros seminais inferiores àqueles propostos pela OMS podem conseguir a gestação (com valores iguais ou superiores a $5 \times 10^{6}$ de formas móveis totais e pelo menos $30 \%$ de formas com motilidade progressiva). Neste mesmo estudo, é ressaltado que parâmetros mínimos para a ocorrência de gestação (em apenas 3,6\% dos casos) ficam ao redor de 2 x $10^{6} / \mathrm{mL}$ de espermatozóides, $17 \%$ de formas $\mathrm{A}+\mathrm{B}$ e motilidade total de $1,6 \times 10^{6} / \mathrm{mL}$. Não houve diferença significativa nas taxas de gestação obtidas no estudo de Huang et al. ${ }^{20}$, quando amostras com mais de $40 \%$ de formas normais foram comparadas com amostras com valores inferiores a $40 \%$ (23,4 vs 24,4\%, respectivamente). Em outros estudos, taxas ao redor de $12 \%$ de gestação foram conseguidas em amostras com motilidade total média de 10 milhões de espermatozóides. No entanto, valores entre 5 a 10 milhões de formas móveis no ejaculado e cerca de 1 milhão de formas móveis inseminadas têm sido relatados em estudos recentes, com boas chances de sucesso ${ }^{10,13,15}$.

De acordo com os parâmetros mínimos para a indicação da técnica de IIU neste estudo (pacientes com concentração de espermatozóides móveis 
progressivos por mL após o processamento de 5 milhões e no mínimo $4 \%$ de formas normais de acordo com o critério estrito de Kruger), foi possível notar que os valores obtidos antes ou depois do processamento relacionados à concentração e à motilidade dos espermatozóides não parecem interferir nos resultados.

Dado importante observado neste trabalho referiu-se ao número de espermatozóides inseminados. Amostras inseminadas contendo menos que $15 \times 10^{6} / \mathrm{mL}$ de espermatozóides acarretaram taxas de gestação significativamente reduzidas. A exemplo do que ocorre na fertilização in vivo, muitos espermatozóides aptos para a fertilização do oócito são necessários para que apenas um consiga finalizar o processo e número aumentado de espermatozóides móveis progressivos parece aumentar as chances de fertilização e conseqüentemente de gestação.

Em consonância com outros estudos ${ }^{21-24}$, foi possivel perceber que a morfologia do espermatozóide representou um parâmetro de suma importância, capaz de diferenciar os casais com maior probabilidade de gravidez. Os pacientes do grupo com gestação positiva apresentaram cerca de $10 \%$ de formas normais, contra média de $6 \%$ naqueles em que a gestação não ocorreu. Neste contexto, Kruger et al. ${ }^{9}$ e Kruger e Coetzee ${ }^{25}$ deixam grande contribuição com a proposta de análise mais criteriosa do espermatozóide. A partir de um estudo dos espermatozóides em diferentes regiões do trato genital feminino, foram sugeridos padrões morfológicos normais para espermatozóides, a exemplo daqueles encontrados na parte superior do canal cervical, sobreviventes e imersos no muco cervical.

Existe forte associação entre alterações morfológicas do espermatozóide e distúrbios moleculares e/ou genéticos, com conseqüente prejuízo do processo de fertilização, desenvolvimento embrionário e gestação. Espermatozóides anormais parecem refletir a ocorrência de dissomias dos cromossomos sexuais e do cromossomo 21 , aneuploidias, alterações estruturais e distúrbios meióticos. Entre eles, as dissomias são as disfunções mais freqüentes nos homens inférteis e as principais responsáveis pelos abortamentos. Estudos citogenéticos de espermatozóides amorfos com cabeça ou cauda duplas demonstraram taxas de $100 \%$ de alteração cromossômica ${ }^{26}$. Uma grande incidência de aberrações cromossômicas parece estar diretamente relacionada a anormalidades morfológicas na cabeça do espermatozóide, interferindo de maneira importante no desenvolvimento embrionário. Estudos sugerem que, quando a morfologia espermática fica entre $0-4 \%$ de formas normais, a taxa de gestação em IIU é de $2,63 \%$ por ciclo, ao passo que em amostras com mais de $14 \%$ de formas normais, ocorre elevação drástica das taxas de gestação (24\%). No geral, sugere-se a indicação para a IIU somente quando pelo menos $4 \%$ dos espermatozóides exibir morfologia normal, de acordo com o critério estrito de Kruger, muito embora taxas de gestação de 5 a $9 \%$ possam ser conseguidas com porcentagens menores de formas normais ${ }^{27}$.

Nossos resultados sugerem que a IIU pode ser realizada com sucesso em pacientes com indicações precisas para este procedimento. Tanto a morfologia espermática quanto o número de espermatozóides transferidos para a cavidade intra-uterina são parâmetros que parecem predizer o sucesso do procedimento, além de distinguir aqueles casais com infertilidade masculina que poderiam se beneficiar com a indicação desta técnica daqueles que deveriam ser encaminhados diretamente para técnicas de alta complexidade.

Acreditamos que, naqueles casos em que a morfologia estrita de Kruger et al. ${ }^{9}$ e Kruger e Coetzee $^{25}$ é superior ou igual a $10 \%$, com possibilidade de se obter amostra final para ser inseminada contendo mais que 15 milhões de formas móveis progressivas, melhor perspectiva em relação à obtenção da gestação em ciclos de IIU pode ser obtida.

\section{ABSTRACT}

Purpose: to analyze the influence of seminal parameters on intrauterine insemination (IUI) outcomes in patients with male factor and to emphasize the predictive value of each parameter for the successful result.

Methods: two hundred and thirty-nine IUI cycles (155 couples) were analyzed for 15 months. Female patients were submitted to ovary hyperstimulation according to the "I Consenso Brasileiro de Indução de Ovulação". Seminal analysis based on the World Health Organization (WHO) for sperm concentration and motility was used and sperm morphology was evaluated according to Kruger's criterion. Samples to be used in IUI were prepared by colloidal discontinued gradient (ISolate ${ }^{\circledR}$ ). After IUI two patient groups were formed: group $G$ - positive for pregnancy and group $N G$ - negative for pregnancy.

Results: there was no statistical difference in total sperm concentration per $m L$, total motility and progressive motility before and after the ISolate ${ }^{\circledR}$ procedure. When sperm morphology was compared between the two groups, a statistical difference was observed (group $G=10.6 \%$ normal morphology; group $N G=6.4 \%$ normal morphology; $p<0.05$ ). Better pregnancy results were obtained when the number of inseminated spermatozoa was more than $15 \times 10^{6} / \mathrm{mL}$.

Conclusions: sperm morphology and the number of inseminated sperm seem to be positive parameters for pregnancy and should be emphasized during male infertility propaedeutics.

KEYWORDS: Infertility. Artificial insemination. Semen analysis. 


\section{Referências}

1. Barrat CL, Cooke ID. Sperm transport in the human female reproductive tract: a dynamic interaction. Int J Androl 1991; 14:394 -411.

2. Borges Júnior E, Iaconelli Júnior A. I Consenso Brasileiro de Indução de Ovulação em Reprodução Assistida. Navio Costa Allegra; 9-12 dez 1999.

3. Oliveira DCA; Borges Júnior E. Reprodução assistida: até onde podemos chegar? Compreendendo a ética e a lei. 1a ed. São Paulo: Gaia; 2000.

4. Wolf DP, Patton PE, Burry KA, Kaplan PF. Intrauterine insemination-ready versus conventional semen cryopreservation for donor insemination: a comparison of retrospective results and a prospective, randomized trial. Fertil Steril 2001; 76:181-5.

5. Aboulghar M, Mansour R, Serour G, Abdrazek A, Amin Y, Rhodes C. Controlled ovarian hyperstimulation and intrauterine insemination for treatment of unexplained infertility should be limited to a maximum of three trials. Fertil Steril 2001; 75:88-91.

6. Hughes EG. The effectiveness of ovulation induction and intrauterine insemination in the treatment of persistent infertility: a meta-analysis. Hum Reprod 1997; 12:1865-72.

7. Goverde AJ, McDonnell J, Vermeiden JP, Schats R, Rutten FF, Schoemaker J. Intrauterine insemination or in-vitro fertilisation in idiophatic subfertility and male subfertility: a randomised trial and cost-effectiveness analysis. Lancet 2000; 355:13-8

8. World Health Organization. Laboratory manual for the examination of human semen and spermcervical mucus interaction. 4th ed. Geneva: WHO; 1999.

9. Kruger TE, Menkveld R, Stander FS, et al. Sperm morphologic features as a prognostic factor in in vitro fertilization. Fertil Steril 1986; 46:1118-23.

10.Campana A, Sakkas D, Stalberg A, et al. Intrauterine insemination: evaluation of the results according to the woman's age, sperm quality, total sperm count per insemination and life table analysis. Hum Reprod 1996; 11:732-6.

11.Hendin BN, Falcone T, Hallak J, et al. Effect of clinical and semen characteristics on efficacy of ovulatory stimulation in patients undergoing intrauterine insemination. J Assist Reprod Genet 2000; 17:189-93.

12.Van Voorhis BJ, Barnett M, Sparks AE, Syrop CH, Rosenthal G, Dawson J. Effect of the total motile sperm count on the efficacy and cost-effectiveness of intrauterine insemination and in vitro fertilization. Fertil Steril 2001; 75:661-8.

13.Dickey RP, Pyrzak R, Lu PY, Taylor SN, Rye PH. Comparison of the sperm quality necessary for successful intrauterine insemination with World Health Organization threshold values for normal sperm. Fertil Steril 1999; 71:684-9.

14.Guzick DS, Carson SA, Coutifaris C, et al. Efficacy of superovulation and intrauterine insemination in the treatment of infertility. N Engl J Med 1999; 340:177-83.

15.Cohlen BJ, te Velde ER, van Kooji RJ, Looman CW, Habbema JD. Controlled ovarian hyperstimulation and intrauterine insemination for treating male subfertility: a controlled study. Hum Reprod 1998; 13:1553-8.

16.Brzechffa PR, Daneshmand S, Buyalos RP. Sequential clomipheme citrate and human menopausal gonadotropin with intrauterine insemination: the effect of patient age on clinical outcome. Hum Reprod 1998; 13:2110-4.

17.Matorras R, Recio V, Corcostegui B, RodriguezEscudero FJ. Recombinant human FSH versus highly purified urinary FSH: a randomized study in intrauterine insemination with husbands' spermatozoa. Hum Reprod 2000; 15:1231-4.

18.Sahakyan M, Harlow BL, Hornstein MD. Influence of age, diagnosis, and cycle number on pregnancy rates with gonadotropin-induced controlled ovarian hyperstimulation and intrauterine insemination. Fertil Steril 1999; 72:500-4.

19.Hughes EG, Collins JA, Gunby J. A randomized controlled trial of three low-dose gonadotrophin protocols for unexplained infertility. Hum Reprod 1998; 13:1527-31.

20.Huang FJ, Chang SY, Lu YJ, Kung FT, Tsai MY, Wu JF. Two different timings of intrauterine insemination for non-male infertility. J Assist Reprod Genet 2000; 17:213-7.

21.Lindheim SR, Barad DH, Zinger M, et al. Abnormal sperm morphology is highly predictive of pregnancy outcome during controlled ovarian hyperstimulation and intrauterine insemination. J Assist Reprod Genet 1996; 13:569-72.

22. Check ML, Bollendorf A, Check JH, Katsoff D. Reevaluation of the clinical importance of evaluating sperm morphology using strict criteria. Arch Androl 2002; 48:1-3.

23.Van Waart J, Kruger TF, Lombard CJ, Ombelet W. Predictive value of normal sperm morphology in intrauterine insemination (IUI): a structured literature review. Hum Reprod Update 2001; 7:495-500.

24.Lee RK, Hou JW, Ho HY, et al. Sperm morphology analysis using strict criteria as a prognostic factor in intrauterine insemination. Int J Androl 2002; 25:277-80.

25.Kruger TF, Coetzee K. The role of sperm morphology in assisted reproduction. Hum Reprod Update 1999; $5: 172-8$.

26.Lee JD, Kamiguchi Y, Yanagimachi R. Analysis of chromosome constitution of human spermatozoa with normal and aberrant head morphologies after injection into mouse oocytes. Hum Reprod 1996; 11:1942-6.

27.Karabinus DS, Gelety TJ. The impact of sperm morphology evaluated by strict criteria on intrauterine insemination success. Fertil Steril 1997; 67:536-41.

Recebido em: 29/10/2003 Aceito com modificações em: 19/5/2003 